\title{
Wie sollte das Deliktsrecht bei beschränkt rationalem Verhalten des Schädigers ausgestaltet sein? - Das Beispiel Abschlussprüferhaftung
}

\section{Zur Prämisse des rationalen Entscheidens in der ökonomischen Analyse des Rechts}

Dieser Beitrag beleuchtet das Deliktsrecht, das einen zentralen Untersuchungsgegenstand der ökonomischen Analyse des Rechts darstellt. ${ }^{1}$ Folgende Fragen werden gestellt:

(1) Was ändert sich an den Aussagen zur ökonomischen Analyse des Deliktsrechts, wenn man beschränkt rationales Verhalten des potentiellen Schädigers im Sinne der Prospect Theory nach Kahneman/Tversky (1979) unterstellt?

(2) Ist dann eher eine Gefährdungshaftung oder eine Verschuldenshaftung anzustreben?

Üblicherweise unterstellt die ökonomische Analyse in ihren vornehmlich normativen Analysen, dass Individuen rational im Sinne der Erwartungsnutzentheorie entscheiden. ${ }^{2}$ Nach dieser Theorie sollte man sich für diejenige Alternative entscheiden, die in der Zukunft im »Mittel« den höchsten Nutzen stiftet. Wohlgemerkt, das ist nicht zwingend die Alternative, die im Mittel den höchsten finanziellen Ertrag erbringt.

Da die Erwartungsnutzentheorie einen primär normativen Anspruch verfolgt, stellt sie gewisse Anforderungen, denen viele Leser ohne Widerwillen zustimmen werden. So sollte der Nutzen einer Entscheidung im Kontext der bisherigen Vermögensposition beurteilt werden. Ob man etwa $100.000 €$ in Aktien der X-AG oder in Bundesanleihen investieren möchte, sollte im Zusammenhang mit der Entwicklung des restlichen Vermögens beurteilt werden. Zudem fordert die Erwartungsnutzentheorie, dass man die Wahrscheinlichkeiten, mit denen man verschiedene künftige Szenarien erwartet, korrekt anwendet. Glaubt man also, dass der Aktienmarkt in einem Jahr mit 30\% Wahrscheinlichkeit einen Kursverfall erlebt, und erwartet man mit 70\% Wahrscheinlichkeit eine Hausse, so sollte man auch mit diesen Werten rechnen. Wohlgemerkt, die Wahrscheinlichkeit muss nicht korrekt geschätzt sein - soviel wird nicht verlangt und kann man auch nicht verlangen. Insgesamt sollten sich die Wahrscheinlichkeiten zu $100 \%$ addieren. Auch dieses Postulat hört sich vernünftig an.

Nun ist es leider so, dass Individuen bei realen Entscheidungen diese Postulate offenbar systematisch verletzen, in anderer Hinsicht aber durchaus rational im Sinne der Erwar-

* Für wertvolle Hinweise danke ich Frau Dr. Anja Schöttner von der Humboldt Universität Berlin und Seminarteilnehmern an den Jahrestagungen der American Law and Economics Association (Berkeley 2006), der European Association of Law and Economics (Laibach 2005) und des Behavioral Law and Economics Workshops, insbesondere Andreas Ortmann, CERGE-El Prag.

1 Siehe Schäfer / Ott (2005).

2 Siehe zur Erwartungsnutzentheorie, die oft auch als Bernoulli-Prinzip bezeichnet wird, Bitz (1981), Kapitel 3. 
tungsnutzentheorie handeln. Man spricht daher auch von beschränkt rationalem Verhalten. Auf diese Befunde deuten zahlreiche Experimente hin, die in der Pionierzeit mit den Namen Amos Tversky, Daniel Kahneman und Vernon Smith verbunden sind, weshalb die letzteren beiden 2002 auch mit dem Nobelpreis für Wirtschaftswissenschaften ausgezeichnet wurden (Amos Tversky war schon verstorben). Kahneman und Tversky haben bereits 1979 ein alternatives Entscheidungskonzept vorgestellt, die so genannte Prospect Theory, die wir in den Kapiteln 3 und 4 ausführlicher vorstellen werden. ${ }^{3}$ Es gibt daneben zahlreiche andere Vorschläge, um »reales« Entscheidungsverhalten konzeptionell abzubilden, doch ist die Prospect Theory das m.E. dominierende deskriptive Entscheidungskonzept in der Wirtschaftswissenschaft. Kaum ein wirtschaftwissenschaftlicher Aufsatz wird so häufig zitiert wie derjenige von Kahneman / Tversky aus dem Jahre 1979.

Im Bereich der ökonomischen Analyse des Rechts sind diese neueren Überlegungen bisher jedoch kaum eingeflossen. ${ }^{4}$ Shavell, der Autor des international wichtigsten Lehrbuchs zur ökonomischen Analyse des Deliktsrechts (Shavell, 1987), geht strikt von der Prämisse der Erwartungsnutzentheorie aus, ebenso in seinem jüngeren Lehrbuch (Shavell, 2004). Der Bereich Behavioral Law and Economics befindet sich in seiner Kindheitsphase. Ein erster Meilenstein stellt der Sammelband von Sunstein (2000) dar, allerdings werden dort längst nicht alle Sachverhalte behandelt, die aus Sicht von Juristen wie auch Ökonomen interessant wären. ${ }^{5}$

Erst in jüngster Zeit nehmen verschiedene Beiträge die Idee beschränkt rationalen Verhaltens in der ökonomischen Analyse des Deliktsrechts auf. Zu nennen sind Eide (2006), dessen Beitrag auch in diesem Tagungsband vertreten ist, und Teitelbaum (2006). Beide Aufsätze unterstellen ebenfalls, dass der Schädiger beschränkt rational handelt, unterstellen jedoch andere Entscheidungskonzepte, die im wissenschaftlichen Schrifttum weniger verbreitet sind als die Prospect Theory, namentlich die Rank Dependent Expected Utility ${ }^{6}$ (RDEU) bzw. die Choquet Expected Utility (CEU). Beide Theorien unterstellen wie die Prospect Theory, dass Individuen die Eintrittswahrscheinlichkeit des künftigen Schadensereignisses nicht korrekt gewichten. Anders als Eide (2006) und Teitelbaum (2006) analysieren wir aber zusätzlich auch die Rolle der Verlustaversion, d.h. die offenbar weit verbreitete Scheu, Verluste eingehen zu wollen. Interessanterweise kommen alle drei Aufsätze zu einer ähnlichen Schlussfolgerung: Ist beschränkt rationales Verhalten der potentiellen Schädiger zu vermuten, sollte der Gesetzgeber eher eine Verschuldens- als eine Gefährdungshaftung vorsehen.

3 Siehe Kahneman / Tversky (1979). Die Prospect Theory wurde 1992 zur so genannten Cumulative Prospect Theory weiterentwickelt, ohne dass sich die wesentlichen Charakteristika änderten, siehe Tversky / Kahneman (1992).

4 In dem im deutschen Sprachraum maßgeblichen Lehrbuch zur ökonomischen Analyse des Rechts von Schäfer / Ott (2005) finden sich nur vereinzelt Passagen, die neuere Entscheidungskonzepte berücksichtigen, gleiches gilt für das international bekannte Lehrbuch von Cooter / Ulen (2004).

5 Der Untersuchungsgegenstand des vorliegenden Beitrags wird ebenfalls nicht behandelt. Inhaltlich am nächsten ist ein Beitrag über «Assessing punitive damages" von Sunstein, Kahneman und Schkade (2004).

6 Siehe zur RDEU etwa Quiggin (1982) und Segal (1987), siehe zur CEU Schmeidler (1989). 

sein?

Die Kapitel 3 und 4 untersuchen nun, welchen Einfluss die beiden noch näher zu erläuternden zentralen Elemente der Prospect Theory - nämlich (1) die Verlustaversion und (2) die fehlerhafte Gewichtung von Wahrscheinlichkeiten - auf den Schadensvermeidungsanreiz des potentiellen Schädigers haben und inwiefern hier Abweichungen zur klassischen ökonomischen Analyse des Rechts bestehen. Das klassische Modell präsentieren wir in Kapital 2. Zudem ist zu fragen, welches Haftungsregime zu Lösungen führt, die aus gesellschaftlicher Sicht wünschenswert sind. Um die Analyse übersichtlich zu halten, wird sie auf den Fall beschränkt, in dem der Schaden alleine durch den Schädiger verursacht wird. Mitverschulden betrachten wir nicht. Als Beispiel ziehen wir die Tätigkeit eines Abschlussprüfers heran. ${ }^{7}$

\section{Das klassische Modell zur ökonomischen Analyse des Deliktsrechts}

Wir betrachten eine börsennotierte Aktiengesellschaft, deren Jahresabschluss zu prüfen ist. Es sei unterstellt, der Abschluss sei fehlerhaft und verstoße gegen gesetzliche oder standesrechtliche Vorgaben. Eine wichtige Aufgabe des Abschlussprüfers ist es dann, diesen Fehler zu entdecken. Wird der Fehler entdeckt und der Abschluss entsprechend korrigiert, treffen (potentielle) Anleger grundsätzlich bessere Entscheidungen. Wird der Fehler jedoch nicht aufgedeckt, investieren Anleger möglicherweise in das Unternehmen, obwohl sie hierzu im Falle der Aufdeckung nicht oder nur zu anderen Konditionen bereit gewesen wären. Ohne auf die Probleme der aus ökonomischer Sicht korrekten Schadensbemessung näher einzugehen, ${ }^{8}$ sei angenommen, dass der Schaden für die Anlegergemeinschaft $D$ betrage, sofern der Abschlussprüfer den Fehler nicht entdeckt. ${ }^{9}$

Der Abschlussprüfer darf keine erfolgsabhängige Entlohnung erhalten, vereinfachenderweise nehmen wir an, dass er ein Pauschalhonorar erhält, welches alle Kosten abdeckt. Zudem wird im klassischen Modell zur ökonomischen Analyse des Deliktsrechts angenommen, dass geschädigte Personen den Schädiger in unbeschränkter Höhe haftbar machen können und dass Anleger immer dann klagen, wenn sie einen Schaden erleiden. Dies entspricht sicherlich eher dem US-amerikanischen Modell der

7 Auch das Schrifttum zur optimalen Haftung des Abschlussprüfers berücksichtigt bisher nicht beschränkt rationales Verhalten, siehe etwa Dye (1993), Schwartz (1997), Ewert (1999), Zhang / Thoman (1999), Hillegeist (1999), Radhakrishnan (1999), Schäfer (2004), Ganuza/Gomez (2005) und Bigus / Schäfer (2007). Im Zentrum stehen dort andere Fragestellungen, etwa die Wirkungen betragsmäßig beschränkter Haftung, außergerichtlicher Einigungen oder Versicherungen auf die Sorgfaltsanreize des Prüfers, das Für und Wider gesamtschuldnerischer versus proportionaler Haftung, und die Rolle des Abschlussprüfers auf Primär- und Sekundärmarkten.

8 Siehe hierzu Bigus / Schäfer (2007).

9 Es sei angemerkt, dass der Schaden der geschädigten Anleger oftmals größer ist als der aus ökonomischer Sicht relevante - Schaden der Anlegergemeinschaft. Dies ist ein generelles Resultat für Vermögensschäden. Dieses Resultat liegt darin begründet, dass es auch Anleger geben kann, die von einem fehlerhaften Testat oder Bilanzfehlern profitieren, da sie etwa Aktien überbewertet verkaufen können, diese Gewinne jedoch nach geltendem Recht nicht auskehren müssen. Siehe hierzu Bigus / Schäfer (2007). 
Abschlussprüferhaftung als dem deutschen. § 323 II HGB sieht keine Dritthaftung ${ }^{10}$ vor, (b) der Schadenersatz ist zudem auf 4 Mio. € betragsmäßig beschränkt. Letztlich sind (c) die prozessrechtlichen Voraussetzungen weniger klagefördernd als in den USA, wo reine Erfolgshonorare vereinbart werden können, Sammelklagen leichter möglich sind und die Aufteilung der Prozesskosten nicht vom Ausgang des Verfahrens abhängt. ${ }^{11}$

Wir bleiben weiter beim Modell der Abschlussprüferhaftung. Der Abschlussprüfer als potentieller Schädiger kann die Wahrscheinlichkeit, den Fehler in der Bilanz zu entdecken, selbst steuern: Je härter er arbeitet - oder technisch gesprochen: je höher sein Schadensvermeidungs- oder Sorgfaltsaufwand ist - desto eher wird er den Bilanzfehler entdecken. Die folgende Graphik verdeutlicht diesen Zusammenhang zwischen dem Sorgfaltsaufwand $x$ und der Wahrscheinlichkeit $p(x)$, den Fehler nicht zu entdecken und daher Schadenersatz zahlen zu müssen.

Abbildung 1: Der Zusammenhang zwischen dem Sorgfaltsaufwand des Abschlussprüfers und der Wahrscheinlichkeit, den Fehler in der Bilanz zu entdecken

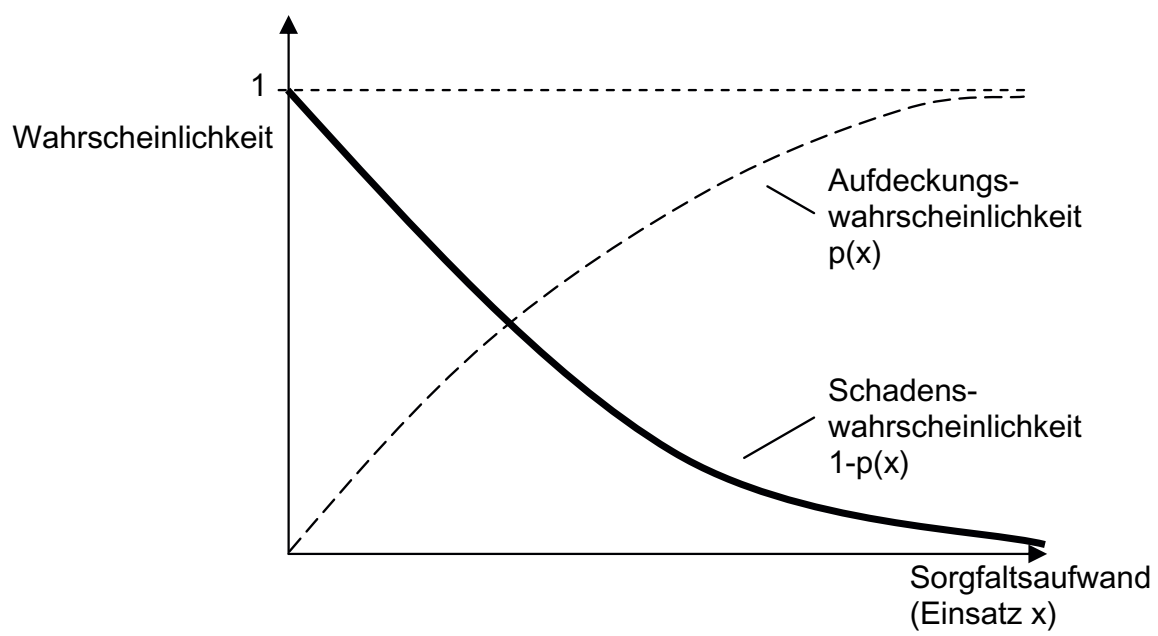

10 Für eine Dritthaftung kommen nur die Generalnormen nach $\S 823$ II und 826 BGB in Frage, allerdings dürfte die Anspruchsvoraussetzung vorsätzlichen Handelns kaum nachweisbar sein. Der BGH hat zudem 1998 festgestellt, dass die Rechtsfigur des »Vertrags mit Schutzwirkung zugunsten Dritter « grundsätzlich auch auf die Abschlussprüferhaftung übertragbar sei, allerdings nur bei Haftungsbeschränkung nach $\S 323$ II HGB und bei enger und genauer Abgrenzung des Kreises betroffener Anleger.

11 Das Grundmodell zur ökonomischen Analyse des Deliktsrechts kann man in Deutschland auf solche Kontexte anwenden, in denen die genannten Voraussetzungen (etwa eine unbeschränkte Haftung) grundsätzlich gegeben sind, z.B. für Delikte im Straßenverkehr oder für Umweltdelikte. 

sein?

Abbildung 1 liegt die Vorstellung zugrunde, dass der Fehler mit Sicherheit ( $p=$ $100 \%=1$ ) nicht entdeckt wird und mit Sicherheit zu haften ist, wenn der Abschlussprüfer überhaupt keine Anstrengung unternimmt $(x=0) .{ }^{12}$ Mit zunehmendem Einsatz, d.h. mit jeder mehr aufgewendeten Arbeitsstunde, steigt die Aufdeckungswahrscheinlichkeit. Allerdings wird der Prüfer nicht mit allerletzter Sicherheit den Fehler entdecken, selbst wenn er noch so hart arbeitet. Daher besteht die Vorstellung, dass der $z u$ sätzliche Nutzen jeder weiteren Arbeitsstunde immer mehr abnimmt. Die 3. Stunde der Prüfung liefert fundamentalere Informationen als die 531. Stunde, in der nur noch wenige zusätzliche Details gewonnen werden können. Dem Vorteil einer zusätzlichen Arbeitsstunde, nämlich die Verringerung der Schadenswahrscheinlichkeiten, steht der Nachteil höherer Prüfkosten gegenüber (die bei einem Fixhonorar nicht vergütet werden).

Man kann diesen Zusammenhang auch an folgendem Zahlenbeispiel konkretisieren. Unterstellt sei, dass jede Arbeitsstunde mit allen Nebenkosten $200 €$ koste. Der Schaden bei nicht aufgedecktem Bilanzfehler betrage $D=1$ Mio. $€$. Der Zusammenhang zwischen Sorgfaltsaufwand und Fehler- und Schadenswahrscheinlichkeit sei durch Tabelle 1 verdeutlicht:

Tabelle 1: Direkte Prüfungskosten, Schadenswahrscheinlichkeit, erwarteter Schaden und Gesamtkosten bei unterschiedlichem Einsatz des Abschlussprüfers

\begin{tabular}{|c|c|c|c|c|c|c|c|}
\hline $\begin{array}{l}\text { (1) Sorgfaltsaufwand } x \text { in } \\
\text { Stunden }\end{array}$ & 400 & 500 & 600 & 700 & 800 & 900 & 1.000 \\
\hline $\begin{array}{l}\text { (2) direkte Prüfungskos- } \\
\text { ten }=(1) \cdot 200 €\end{array}$ & 80.000 & 100.000 & 120.000 & 140.000 & 160.000 & 180.000 & 200.000 \\
\hline $\begin{array}{l}\text { (3) Fehler- und Schadens- } \\
\text { wahrscheinlichkeit }\end{array}$ & $12 \%$ & $9 \%$ & $6,5 \%$ & $5 \%$ & $4 \%$ & $3,3 \%$ & $2,8 \%$ \\
\hline $\begin{array}{l}\text { (4) Erwarteter Schaden } \\
\text { im Zeitpunkt der Prü- } \\
\text { fung }=(3) \cdot 1 \text { Mio. } €\end{array}$ & 120.000 & 90.000 & 65.000 & 50.000 & 40.000 & 33.000 & 28.000 \\
\hline $\begin{array}{l}\text { (5) Gesamtkosten }= \\
(2)+(4)\end{array}$ & 200.000 & 190.000 & 185.000 & 190.000 & 200.000 & 213.000 & 228.000 \\
\hline
\end{tabular}

Die Gesamtkosten der Prüfungstätigkeit umfassen die (direkten) Kosten der Abschlussprüfung und die zu erwartenden Schäden. Der zu erwartete Schaden entspricht

12 Beachtet man die Haftungsbeschränkung nach § 323 II HGB und die im Vergleich zu den USA gedämpften Klageanreize und auch Beweisschwierigkeiten, dürfte selbst bei einem Nulleinsatz nicht mit Sicherheit von einer Haftung auszugehen sein, wie es Abbildung 1 suggeriert. Dies ist aber hier nicht wichtig: bedeutsam ist nur der Zusammenhang, dass der Prüfer umso eher den Fehler entdeckt und umso weniger haftet, je höher sein Sorgfaltsaufwand ist. 
dem Produkt aus dem Schaden der Anlegergemeinschaft im Falle eines Bilanzfehlers und der Wahrscheinlichkeit, dass dieser Bilanzfehler nicht vom Prüfer entdeckt wird.

Was sagt nun die ökonomische Analyse des Rechts? Die Prüfungstätigkeit soll nicht uferlos ausgeführt werden, sondern nur bis zu dem Punkt, bis zu dem der zusätzlich zu erwartende Nutzen die zusätzlichen Kosten übersteigt. Dies ist offenbar für 600 Prüfungsstunden gegeben. Wählt ein Prüfer von 500 Stunden ausgehend, 100 Stunden mehr, so kostet dies zusätzlich $20.000 €$, die über das Fixgehalt nicht zusätzlich vergütet werden, jedoch werden ein Bilanzfehler und eine Schadenersatzzahlung auch unwahrscheinlicher (von 9\% auf 6,5\%). Der zusätzliche Nutzen entspricht daher 2,5\%·(1 Mio. $€)=25.000 €$ und übersteigt die direkten Prüfungskosten. Der Übergang von 600 auf 700 Stunden lohnt sich indes nicht, den zusätzlichen Prüfungskosten von $20.000 €$ steht ein zusätzlicher Nutzen von $(6,5 \%-5 \%) \cdot 1$ Mio. $€=15.000 €$ gegenüber. Die Gesamtkosten sind daher - im Beispiel - für ein Arbeitsvolumen von 600 Stunden am geringsten und betragen $185.000 €$.

Das ist eine wichtige Nachricht gerade in Zeiten gehäufter Bilanzskandale, in denen das richtige Augenmaß zuweilen verloren geht: ein potentieller Schaden sollte nicht um jeden Preis vermieden werden. ${ }^{13}$ Dies ist ökonomisch nicht wünschenswert, auch nicht aus Anlegersicht, da der Prüfer letztendlich diese Kosten über die Höhe des Prüfungshonorars auf die Anleger abwälzt.

Die Frage ist nun, ob Verschuldens- oder Gefährdungshaftung den Abschlussprüfer dazu bewegen, diese 600 Stunden, die aus gesellschaftlicher Sicht das Optimum darstellen, auch wirklich abzuleisten. Der Prüfer wählt bei einem Fixhonorar immer das Sorgfaltsniveau, das unter Berücksichtigung der direkten Prüfungskosten und der erwarteten Schadenersatzzahlungen minimal ist. Gäbe es kein Haftungsregime, so ist offensichtlich, dass der Prüfer den geringstmöglichen Sorgfaltsaufwand erbringen würde, sofern nicht andere Sanktionsmechanismen greifen (z.B. strafrechtliche Konsequenzen oder Reputationsverluste $\left.{ }^{14}\right)$. Denn dann entfallen die zu erwartenden Schadenersatzzahlungen aus Zeile (4).

Bei Gefährdungshaftung haftet der Abschlussprüfer idealer Weise stets dann, wenn ein Schaden auftritt - unabhängig von seinem Sorgfaltsaufwand. Damit trägt er stets den Schadenersatz und zudem auch die direkten Kosten der Prüfung, d.h. die Gesamtkosten. Der Prüfer arbeitet daher bei Gefährdungshaftung 600 Arbeitsstunden, was auch im Interesse der Aktionäre ist. ${ }^{15}$

13 Die Gefahr übermäßiger Sorgfalt scheint eher in den USA als in Deutschland gegeben zu sein.

14 Zur Rolle von Reputationsverlusten im Kontext der Abschlussprüferhaftung siehe Bigus (2006a).

15 Wir sehen hier von Beweisschwierigkeiten und beschränktem Haftungsvermögen ab. Je mehr die Beweislast auf Seiten der Anleger liegt und je schwieriger und anspruchsvoller die Beweisführung ist, je geringer die Sachkenntnis und/oder die Unabhängigkeit der Gerichte ist, je geringer das tatsächliche Vermögen des Prüfers ist und je umfangreicher die Dekkungssumme einer Versicherungsgesellschaft ist, desto weniger wird ein Prüfer ceteris paribus geneigt sein, gerade die wünschenswerten 600 Stunden zu erbringen. Ähnliches gilt prinzipiell auch für die Verschuldenshaftung. 

sein?

Bei Verschuldenshaftung haftet der Prüfer nur dann, wenn ein Schaden eintritt und er einen gesetzlich definierten Sorgfaltsstandard nicht eingehalten hat, d.h. die im Verkehr erforderliche Sorgfalt fahrlässig verletzt hat. Die Grundsätze ordnungsgemäßer Abschlussprüfung sind in gesetzlichen wie auch standesrechtlichen Regelungen präzisiert, etwa in der Wirtschaftsprüferordnung. Entspricht der gesetzliche Standard nun gerade dem gesellschaftlich optimalem, d.h. impliziert die Einhaltung der Regelungen bei diesem Unternehmen gerade 600 Arbeitsstunden, so wird der Wirtschaftsprüfer diese 600 Stunden auch arbeiten, da seine Kosten dann minimal sind (und $120.000 €$ betragen). Wählt er einen geringeren Sorgfaltsaufwand, so muss er zusätzlich bei einem Schaden haften. Die Gesamtkosten sind dann für ihn höher, wie Tabelle 2 verdeutlicht.

Tabelle 2: Optimaler Sorgfaltsaufwand des Abschlussprüfers ohne Haftungsregime, bei Gefährdungs- und bei Verschuldenshaftung (Fettdruck zeigt das Kostenminimum an)

\begin{tabular}{|c|c|c|c|c|c|c|c|}
\hline $\begin{array}{l}\text { (1) Sorgfaltsaufwand } x \text { in } \\
\text { Stunden }\end{array}$ & 400 & 500 & 600 & 700 & 800 & 900 & 1.000 \\
\hline $\begin{array}{l}\text { (2) direkte Kosten }= \\
\text { (1) } \cdot 200 €\end{array}$ & 80.000 & 100.000 & 120.000 & 140.000 & 160.000 & 180.000 & 200.000 \\
\hline $\begin{array}{l}\text { (3) Fehler- und Schadens- } \\
\text { wahrscheinlichkeit }\end{array}$ & $12 \%$ & $9 \%$ & $6,5 \%$ & $5 \%$ & $4 \%$ & $3,3 \%$ & $2,8 \%$ \\
\hline $\begin{array}{l}\text { (4) Erwarteter Schaden } \\
\text { im Zeitpunkt der } \\
\text { Prüfung }= \\
(3) \cdot 1 \text { Mio. } €\end{array}$ & 120.000 & 90.000 & 65.000 & 50.000 & 40.000 & 33.000 & 28.000 \\
\hline $\begin{array}{l}\text { (5) Gesamtkosten = } \\
(2)+(4)\end{array}$ & 200.000 & 190.000 & 185.000 & 190.000 & 200.000 & 213.000 & 228.000 \\
\hline $\begin{array}{l}\text { Kosten des Prüfers ohne } \\
\text { Haftungsregime = }(2)\end{array}$ & 80.000 & 100.000 & 120.000 & 140.000 & 160.000 & 180.000 & 200.000 \\
\hline $\begin{array}{l}\text { Kosten des Prüfers bei } \\
\text { Gefährdungshaftung= } \\
\text { (5) }\end{array}$ & 200.000 & 190.000 & 185.000 & 190.000 & 200.000 & 213.000 & 228.000 \\
\hline $\begin{array}{l}\text { Kosten des Prüfers bei } \\
\text { Verschuldenshaftung } \\
\text { mit Sorgfaltsstandard } x^{S}= \\
600 \mathrm{~h}\end{array}$ & 200.000 & 190.000 & 120.000 & 140.000 & 160.000 & 180.000 & 200.000 \\
\hline
\end{tabular}

Die Tabelle zeigt ein generelles Ergebnis der ökonomischen Analyse des Deliktsrechts: Eine Gefährdungs- wie auch eine korrekt spezifizierte Verschuldenshaftung vermitteln bei unilateralen Schäden optimale Sorgfaltsanreize. Ohne Haftungsregime 
wird der Abschlussprüfer nachlässig prüfen, diesen Fall werden wir daher im Weiteren nicht betrachten. Eine falsch spezifizierte Verschuldenshaftung (in unserem Beispiel: $x^{S}<600$ h oder $x^{S}>600$ h) führt regelmäßig entweder zu Schlendrian oder zu übermäBiger Sorgfalt. Beides ist nicht wünschenswert. Wegen der Fehleranfälligkeit der Verschuldenshaftung fragt man sich, weshalb die Abschlussprüferhaftung in nahezu sämtlichen westlichen Rechtsordnungen auf Verschulden beruht. ${ }^{16}$ Liegt es daran, dass beschränkt rationales Verhalten der potentiellen Schädiger eine Verschuldensregel rechtfertigt? Diese Frage wollen wir nun im Folgenden beantworten.

\section{Verlustaversion des Abschlussprüfers}

Nun verlassen wir das klassische Modell der ökonomischen Analyse des Rechts und unterstellen beschränkt rationales Verhalten im Sinne der Prospect Theory. Die Prospect Theory weist vier wichtige Elemente auf, die man in zahlreichen Experimenten beobachten konnte. Drei Elemente werden hier und das vierte ein Kapitel später dargestellt.

\section{a. Referenzpunktabhängige Bewertung}

Menschen bewerten unsichere Vermögenspositionen gerade nicht im Zusammenhang mit dem gesamten restlichen Vermögen - wie es die Erwartungsnutzentheorie fordert sondern für sich alleine und im Vergleich zu einem Referenzpunkt. Hierzu ein Beispiel. Herr Muster hat am 2. Januar sein gesamtes Vermögen in den Erwerb der Aktien A und B investiert. Der Kaufpreis war $200 €$ bzw. $300 €$. Am 1. Juli sinkt der Kurs der Aktie A auf $120 €$, derjenige der Aktie B steigt auf $380 €$. Nach der Erwartungsnutzentheorie betrachtet Herr Muster sein Gesamtvermögen und stellt fest, dass sich dieses nicht geändert hat: es beträgt weiterhin $500 €$. Nach der Prospect Theory bewertet er jedoch Aktie A für sich selbst und auch Aktie B für sich selbst. Die Frage ist nun: Was ist der Vergleichsmaßstab (Referenzpunkt) für die Bewertung? Hierzu geben leider weder die Prospect Theory noch die empirische Evidenz eine eindeutige Antwort: Der Referenzpunkt kann der Einstandspreis sein oder auch der Wert, der sich bei einer alternativen Anlage in Aktie C oder in Anleihe D zum 1. Juli ergeben hätte oder auch ein ganz anderer Wert. Wäre es der Einstandspreis, stellte Herr Muster einen Verlust von $80 €$ für Aktie A und einen Gewinn von $80 €$ für Aktie B fest. ${ }^{17}$

16 Man muss dazu sagen, dass eine Verschuldenshaftung bei Mitverschulden (etwa des Managements) aus ökonomischer Sicht Vorzüge gegenüber einer Gefährdungshaftung aufweist. Diese blenden wir hier aus, siehe Schäfer /Ott (2005), S. 221-258.

17 Hätte sich eine Anlage von $200 €$ bzw. $300 €$ in eine festverzinsliche Anlage zu 10\% verzinst und wäre dies der Vergleichsmaßstab, wären die Referenzpunkte $220 €$ bzw. $330 €$ und man rechnete mit einem Verlust von $100 €$ bei Aktie A und einem Gewinn von $50 €$ bei Aktie B. Wir behalten als Referenzmaßstab den Einstandspreis bei. 

sein?

\section{b. Verlustaversion}

Nach Kahneman / Tversky (1979) werden Verluste in ihrer Wirkung stärker wahrgenommen als Gewinne in betragsmäßig gleicher Höhe. Tritt ein Verlust mit einer Vermögensposition auf, so kann dies nur durch einen zwei bis drei Mal so hohen Gewinn einer anderen Vermögensposition kompensiert werden. In unserem Beispiel würde Herr Muster seine Vermögensposition am 1. Juli schlechter bewerten als am 2. Januar. Dem Verlust von $80 €$ bei der Aktie A steht nur ein Gewinn von $80 €$ bei Aktie B gegenüber. Der Verlust zählt aber zwei- bis dreimal soviel, wie empirische Studien zeigen. ${ }^{18}$ Dies wird auch als Verlustaversion bezeichnet.

\section{c. Risikofreudiges Verhalten bei Verlusten, risikoscheues Verhalten bei Gewinnen}

Die Angst, Verluste zu realisieren, führt manchmal dazu, dass Anleger ein Wertpapier nicht verkaufen, obwohl sie glauben, dass sich der Kurs im Durchschnitt in der Zukunft nicht erholen wird, sie hoffen es aber. Dieses Verhalten ist vergleichbar mit demjenigen eines Spielers, der weiß, dass er im Durchschnitt gegen die Spielbank verlieren wird, aber dennoch hofft, gewinnen zu können, und zeigt somit Risikofreude an. ${ }^{19}$ Im Gewinnbereich zeigen Individuen stattdessen risikoscheues Verhalten, d.h. Gewinne werden durch Verkauf der Aktien realisiert, auch wenn man im Durchschnitt noch mit weiteren Kurssteigerungen rechnet.

Die Frage ist nun, wie man Verlustaversion im Kontext der Prüferhaftung konkretisiert. ${ }^{20}$ Dazu ist zunächst der Referenzpunkt zu bestimmen. Es gibt zwei Möglichkeiten.

a) Der Referenzpunkt stellt die Situation ohne Prüfung dar. Dann wären sowohl die direkten Prüfungskosten als auch die künftige Schadenersatzzahlung als Verlust für den Prüfer anzusehen. Der Gewinn wäre das fixe Honorar, das ohnehin unabhängig von dem Sorgfaltsaufwand ist und daher nicht weiter betrachtet werden muss. ${ }^{21}$

b) Nur die Schadenersatzzahlungen werden als Verlust angesehen.

Tabelle 3 zeigt die Konsequenzen für die Fälle a) und b) unter der Annahme auf, dass Verluste 2,5-fach bewertet werden.

18 Tversky / Kahneman (1992), S. 311f. schätzen den Faktor auf Basis eines Experiments auf ca. 2,25. Allerdings variiert der Faktor abhängig davon, was man verlieren kann (Geld, Güter) und in welchem Umfang.

19 Erfährt der Spieler jedoch einen persönlichen Nutzen (Spielleidenschaft), kann das Spielen aus individueller Sicht sogar ökonomisch rational sein.

20 Risikofreude im Verlustbereich und Risikoscheu im Gewinnbereich werde ich nicht weiter behandeln, um den Beitrag noch lesbar zu halten. Siehe hierzu Bigus (2006b).

21 Das Fixhonorar wird jedoch tendenziell umso höher sein, je höher der Grad an Verlustaversion ist, da hiermit auch die Kompensationsforderung ansteigt. 
Tabelle 3: Optimales Sorgfaltsniveau des Abschlussprüfers bei Verlustaversion (Fettdruck zeigt das Kostenminimum an)

\begin{tabular}{|c|c|c|c|c|c|c|c|}
\hline $\begin{array}{l}\text { (1) Sorgfaltsaufwand } \\
x \text { in } \mathrm{h}\end{array}$ & 400 & 500 & 600 & 700 & 800 & 900 & 1.000 \\
\hline $\begin{array}{l}\text { (2) Bewertung direkter } \\
\text { Prüfungskosten } \\
\text { (2a): }(1) \cdot 200 € \cdot 2,5 \\
\text { (2b): }(1) \cdot 200 €\end{array}$ & $\begin{array}{c}200.000 \\
80.000\end{array}$ & $\begin{array}{l}250.000 \\
100.000\end{array}$ & $\begin{array}{l}300.000 \\
120.000\end{array}$ & $\begin{array}{l}350.000 \\
140.000\end{array}$ & $\begin{array}{l}400.000 \\
160.000\end{array}$ & $\begin{array}{l}450.000 \\
180.000\end{array}$ & $\begin{array}{l}500.000 \\
200.000\end{array}$ \\
\hline $\begin{array}{l}\text { (3) Fehler- und Scha- } \\
\text { denswahrscheinlich- } \\
\text { keit }\end{array}$ & $12 \%$ & $9 \%$ & $6,5 \%$ & $5 \%$ & $4 \%$ & $3,3 \%$ & $2,8 \%$ \\
\hline $\begin{array}{l}\text { (4) Bewertung des erwar- } \\
\text { teten Schadens = (3) } \\
1 \text { Mio. } € \cdot 2,5\end{array}$ & 300.000 & 225.000 & 162.500 & 125.000 & 100.000 & 82.500 & 70.000 \\
\hline $\begin{array}{l}\text { Kosten des Prüfers bei } \\
\text { Gefährdungshaftung } \\
\qquad \begin{array}{l}(5 a)=(2 a)+(4) \\
(5 b)=(2 b)+(4)\end{array}\end{array}$ & $\begin{array}{l}500.000 \\
380.000\end{array}$ & $\begin{array}{l}475.000 \\
325.000\end{array}$ & $\begin{array}{l}\mathbf{4 6 2 . 5 0 0} \\
282.500\end{array}$ & $\begin{array}{l}475.000 \\
265.000\end{array}$ & $\begin{array}{l}500.000 \\
\mathbf{2 6 0 . 0 0 0}\end{array}$ & $\begin{array}{l}532.500 \\
262.500\end{array}$ & $\begin{array}{l}570.000 \\
270.000\end{array}$ \\
\hline $\begin{array}{l}\text { Kosten bei Verschulden- } \\
\text { shaftung mit Standard } \\
x^{S}=600 \mathrm{~h} \\
\text { bei a) } \\
\text { bei b) }\end{array}$ & $\begin{array}{l}500.000 \\
380.000\end{array}$ & $\begin{array}{l}475.000 \\
325.000\end{array}$ & $\begin{array}{l}300.000 \\
120.000\end{array}$ & $\begin{array}{l}350.000 \\
140.000\end{array}$ & $\begin{array}{l}400.000 \\
160.000\end{array}$ & $\begin{array}{l}450.000 \\
180.000\end{array}$ & $\begin{array}{l}500.000 \\
200.000\end{array}$ \\
\hline
\end{tabular}

Wir erhalten folgende Ergebnisse, die einer generellen, analytischen Prüfung standhalten: ${ }^{22}$

Fall (a):Werden sowohl Sorgfaltsaufwand als auch potentielle Schadenersatzzahlungen als Verlust angesehen, so führen sowohl die Gefährdungs- als auch eine korrekt spezifizierte Verschuldenshaftung dazu, dass der Schädiger, hier der Abschlussprüfer, eine Sorgfalt aufwendet, die dem gesellschaftlich wünschenswerten Niveau entspricht (im Beispiel: 600 Arbeitsstunden).

Fall (b): Werden lediglich die Schadenersatzzahlungen als Verlust angesehen, so gibt es stärkere Anreize des Prüfers, Schaden zu vermeiden. Der potentielle Vermögensabfluss wiegt nun schwerer als der Einsatz zusätzlicher Arbeit. Daher wird der Prüfer bei der Gefährdungshaftung zwingend über das gesellschaftlich wünschenswerte Ziel hinausschießen, wie auch das Beispiel zeigt (hier wählt der Prüfer einen Einsatz von 800h). Bei der Verschuldenshaftung gibt es ebenfalls eine Tendenz hierzu, allerdings tritt dieser Fall nicht zwingend ein, im Beispiel wird der Prüfer etwa den optimalen Einsatz von $600 \mathrm{~h}$ erbringen. Wird der gesetzliche Sorgfaltsstandard

22 Siehe Bigus (2006b). 

sein?

eingehalten oder überschritten, haftet der Prüfer ohnehin nicht. Damit kann auch kein Verlust auftreten. Wie dieser gewichtet wird, ist dann irrelevant.

Diese Analyse zeigt mehrere bedeutsame Ergebnisse. Ist der Gesetzgeber der Ansicht, dass potentielle Schädiger überwiegend verlustavers sind, sollte er ceteris paribus eher eine Verschuldenshaftung anstreben, um die Schädiger von übermäßigem Schadensvermeidungsaufwand abzuhalten. Im Fall (a) sind beide Haftungsregimes jedoch äquivalent. Leider gibt es m.E. bisher keine empirischen Studien, die uns sagen können, ob eher Fall (a) oder Fall (b) in der Praxis relevant ist. Bei Anwendung der klassischen Erwartungsnutzentheorie erhalten wir ähnliche Ergebnisse wie im Fall (b), der Fall (a) ist dort jedoch nicht möglich. ${ }^{23}$

\section{Gewichtung von Wahrscheinlichkeiten}

\subsection{Illustration des »Sicherheitseffekts«}

Der Nobelpreisträger Allais $(1953)^{24}$ hatte seine Kollegen frühzeitig darauf hingewiesen, dass Menschen nicht immer nach den Postulaten der Erwartungsnutzentheorie Entscheidungen treffen. Eines seiner berühmten Paradoxa ist das folgende. Nehmen Sie an, Sie können kostenlos ${ }^{25}$ zwischen zwei Lotterien X und Y wählen. Sie wissen, dass Lotterie X Ihnen mit Sicherheit $(\mathrm{p}=100 \%=1)$ eine Gewinnzahlung von 1 Mio. $€$ garantiert. Lotterie Y führt mit einer Wahrscheinlichkeit von $10 \%$ und $89 \%$ zu einer Zahlung von 5 Mio. $€$ bzw. 1 Mio. $€$, und mit $1 \%$ Wahrscheinlichkeit gibt es keine Gewinnzahlung.

$\mathrm{X}$ : mit $\mathrm{p}=1$ Gewinn von 1 Mio. GE, $\mathrm{Y}:\left\{\begin{array}{l}\text { mit } \mathrm{p}=10 \% \text { gewinnt man } 5 \text { Mio. } € \\ \text { mit } \mathrm{p}=89 \% \text { gewinnt man } 1 \text { Mio. } € \\ \text { mit } \mathrm{p}=1 \% \text { gewinnt man nichts. }\end{array}\right.$

Allais vermutete, dass die meisten Entscheidungssubjekte Alternative X vorziehen würden, weil man hier mit Sicherheit 1 Mio. € vereinnahmen kann. Zwar kann man bei Alternative Y mit $99 \%$ unter Umständen noch mehr erzielen, aber die Differenz von einem Prozentpunkt zählt viel. Die Vermutung von Allais wurde durch nachfolgende empirische Studien bestätigt. ${ }^{26}$

23 Siehe Shavell (1987), S. 206-237. Unser Beitrag beachtet nicht die Fragestellung der optimalen Risikoteilung. Kann man etwa davon ausgehen, dass die potentiell Geschädigten - in unserem Beispiel die Anleger - nicht verlustavers sind oder einen geringeren Grad an Verlustaversion als der Prüfer aufweisen, ist eine Verschuldenshaftung auch aus Gründen der optimalen Risikoteilung vorziehenswürdig. Bei Gefährdungshaftung trägt der Prüfer nämlich sämtliche Schadensrisiken. In der Finanzierungstheorie herrscht die Auffassung vor, dass Anleger besser als Wirtschaftsprüfer in der Lage seien, Vermögensrisiken zu diversifizieren und daher insgesamt weniger verlustavers sein sollten.

24 Siehe Allais (1953), S. 526f.

25 Das Beispiel funktioniert auch, wenn Sie einen Einsatz für die Lotterie zahlen müssen, sofern dieser für beide Lotterien gleich hoch ist und Sie nicht davon abhält, an der Lotterie teilzunehmen. 
Subtrahiert man nun von $\mathrm{X}$ und $\mathrm{Y}$ in gleicher Weise die Möglichkeit, mit einer Wahrscheinlichkeit von 89\% 1 Mio. € zu gewinnen, so ergeben sich die Alternativen $\mathrm{X}^{\prime}$ bzw. Y':

$X^{\prime}:\left\{\begin{array}{l}\text { mit } \mathrm{p}=11 \% \text { gewinnt man } 1 \text { Mio. } €, \\ \text { mit } \mathrm{p}=89 \% \text { gewinnt man nichts. }\end{array} \mathrm{Y}^{\prime}:\left\{\begin{array}{l}\text { mit } \mathrm{p}=10 \% \text { gewinnt man } 5 \text { Mio. } € \\ \text { mit } \mathrm{p}=90 \% \text { gewinnt man nichts. }\end{array}\right.\right.$

Allais vermutete, dass nun die meisten Entscheidungssubjekte $\mathrm{Y}^{\prime}$ vorziehen würden. Präferiert ein Entscheidungssubjekt die Alternative X gegenüber Y, so sollte es aus normativer Sicht auch die Alternative $X^{\prime}$ gegenüber $Y^{\prime}$ bevorzugen. Allais behauptete jedoch, dass die meisten Personen die Alternative $\mathrm{X}$ gegenüber $\mathrm{Y}$ präferieren, jedoch andererseits $\mathrm{X}^{\prime}$ gegenüber $\mathrm{Y}^{\prime}$ bevorzugen. Empirische Studien bestätigen dies.

Der Grund für die Präferenzumkehr in dem Beispiel liegt darin begründet, dass die Differenz zwischen 99\% (Fast-Sicherheit) und 100\% (Sicherheit) offenbar als größer empfunden wird als diejenige zwischen $10 \%$ und $11 \%$. Individuen scheinen Sicherheit (100\% Wahrscheinlichkeit) besonders hoch zu schätzen, daher spricht man auch vom Sicherheitseffekt. Der Sprung von 99\% auf 100\% entspricht in der Wertschätzung der meisten Individuen mehr als einem Prozentpunkt. Sehr große Wahrscheinlichkeiten, hier die $99 \%$, werden untergewichtet.

\subsection{Der Effekt der Wahrscheinlichkeitsgewichtungsfunktion auf die Sorgfaltsanreize des Abschlussprüfers bei präzise definiertem Sorgfaltsstandard}

Kahneman/Tversky (1979) schlagen daher ein viertes Element der Prospect Theory vor: die Wahrscheinlichkeitsgewichtungsfunktion. Danach verwenden Entscheidungssubjekte nicht die gegebenen Wahrscheinlichkeiten $p$ (in unserem Beispiel die 99\%), sondern stattdessen einen Platzhalter, den Kahneman/Tversky Wahrscheinlichkeits gewicht $\omega(p)$ nennen. ${ }^{27}$ D.h., Kahneman/Tversky gehen davon aus, dass Individuen mit anderen Gewichten rechnen, obwohl ihnen die »richtigen « Wahrscheinlichkeiten vorliegen. Dies ist im Rahmen der Erwatungsnutzentheorie nicht möglich.

Die Wahrscheinlichkeitsgewichtungsfunktion nach Kahneman/Tversky weist folgende wesentlichen Charakteristika auf: ${ }^{28}$

(a) Je höher die originäre Wahrscheinlichkeit $p$ ist, desto höher ist das beigemessene Gewicht $\omega(p)$. Bei Sicherheit sind originäre Wahrscheinlichkeit und Gewicht deckungsgleich: $\omega(p=1)=1$ and $\omega(p=0)=0$.

26 Vgl. etwa McCrimmon/Larsson (1979).

27 Allais (1953), S. 508, 513 hat bereits die Idee der Wahrscheinlichkeitsgewichte aufgeworfen, allerdings nicht formalisiert.

28 Siehe Kahneman/Tversky (1979), S. 280-284. Daneben werden andere Merkmale genannt (die für die ökonomische Analyse der Prüferhaftung nicht bedeutsam erscheinen), etwa dass die Wahrscheinlichkeiten zweier sich ausschließender Ereignisse sich nicht zu 100\% addierten (Subsicherheit). Es gibt verschiedene Vorschläge im Schrifttum, Gewichtungsfunktion formal zu präzisieren, etwa bei Tversky/Kahneman (1992), S. 309 und Prelec (1998). 

sein?

(b) An den Stellen $p=0$ und $p=1$ weist die Gewichtungsfunktion «Sprungstellen $«$ auf. ${ }^{29}$ Diese Sprungstellen spiegeln den Sicherheitseffekt wider.

(c) (Sehr) kleine Wahrscheinlichkeiten werden überschätzt, (sehr) große Wahrscheinlichkeiten hingegen unterschätzt: $\omega(p)>p$ bzw. $\omega(p)<p$.

Abb. 2 zeigt die Gewichtungsfunktion (gestrichelte Linie) im Vergleich zur originären Wahrscheinlichkeit (durchgezogene Linie).

Abb. 2: Wahrscheinlichkeitsgewichtungsfunktion $\omega(p)$ nach der Prospect Theory

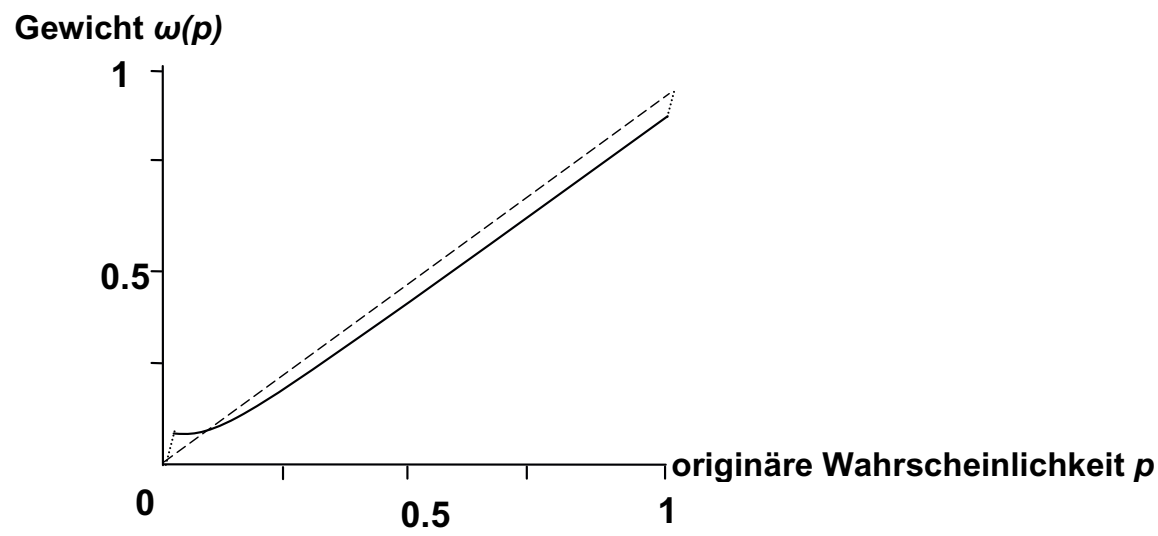

Was bedeutet dies nun für die Anreizwirkung von Haftungsregeln? Zwei Beobachtungen scheinen wichtig zu sein: (1) Wenn eine Haftungsregel »Sicherheit« geben kann, dann wird dies als besonders wertvoll empfunden. Eine Verschuldenshaftung gibt die Sicherheit, keinen Schadenersatz zahlen zu müssen, sofern der gesetzliche Standard eingehalten wird. Die Gefährdungshaftung kann niemals Sicherheit geben, es gibt immer eine (sehr) geringe Wahrscheinlichkeit, zur Haftung herangezogen zu werden. (2) Da kleine Wahrscheinlichkeiten überschätzt, große aber unterschätzt werden, werden die Übergänge dazwischen offenbar unterschätzt. Wird etwa einer Wahrscheinlichkeit von $10 \%$ ein Gewicht von $18 \%$ zugewiesen, einer Wahrscheinlichkeit von $95 \%$ aber ein Gewicht von $88 \%$, dann entspricht die »gefühlte « Steigerung der Wahrscheinlichkeit nur 70 Prozentpunkte (88\% - 18\%), nicht jedoch 85 (95\% - 10\%), wie es rational wäre. Diese Unterschätzung trifft natürlich auch in der Gegenrichtung zu, wenn sich Wahrscheinlichkeiten verringern. Auch dieser Effekt ist bedeutsam, da er dazu führt, dass der Nutzen zusätzlicher Sorgfaltsanstrengungen, nämlich die Verringerung der Haftungswahrscheinlichkeit, unterschätzt wird. Wir wollen diesen Effekt anhand unseres Beispiels verdeutlichen (um diesen Effekt zu isolieren, verzichten wir auf die Verlustaversion).

29 Siehe Kahneman and Tversky (1979), S. 282. 
Tabelle 4: Optimaler Sorgfaltsaufwand des Abschlussprüfers bei Gewichtung der Wahrscheinlichkeiten (Fettdruck zeigt das Kostenminimum an)

\begin{tabular}{|c|c|c|c|c|c|c|c|}
\hline $\begin{array}{l}\text { (1) Sorgfaltsaufwand } x \text { in } \\
\text { Stunden }\end{array}$ & 400 & 500 & 600 & 700 & 800 & 900 & 1.000 \\
\hline (2) direkte Kosten & 80.000 & 100.000 & 120.000 & 140.000 & 160.000 & 180.000 & 200.000 \\
\hline $\begin{array}{l}\text { (3) Fehler- und } \\
\text { Schadenswahr- } \\
\text { scheinlichkeit }\end{array}$ & $12 \%$ & $9 \%$ & $6,5 \%$ & $5 \%$ & $4 \%$ & $3,3 \%$ & $2,8 \%$ \\
\hline $\begin{array}{l}\text { (3a) Gewicht der } \\
\text { Schadenswahr- } \\
\text { scheinlichkeit }\end{array}$ & $12,7 \%$ & $10,4 \%$ & $8,5 \%$ & $7,5 \%$ & $6,7 \%$ & $6,2 \%$ & $6 \%$ \\
\hline $\begin{array}{l}\text { (4) Erwarteter Schaden } \\
\text { im Zeitpunkt der } \\
\text { Prüfung }\end{array}$ & 120.000 & 90.000 & 65.000 & 50.000 & 40.000 & 33.000 & 28.000 \\
\hline $\begin{array}{l}\text { (4a) Gewichteter erwarte- } \\
\text { ter Schaden }=(3 a) \cdot 1 \\
\text { Mio. } €\end{array}$ & 127.000 & 104.000 & 85.000 & 75.000 & 67.000 & 62.000 & 60.000 \\
\hline $\begin{array}{l}\text { (5) Kosten des Prüfers } \\
\text { bei Gefährdungs- } \\
\text { haftung }=(2)+(4 a)\end{array}$ & 207.000 & 204.000 & 205.000 & 215.000 & 227.000 & 242.000 & 260.000 \\
\hline $\begin{array}{l}\text { (6) Kosten des Prüfers } \\
\text { bei Verschuldens- } \\
\text { haftung, mit Sorg- } \\
\text { faltsstandard } \\
x^{S}=600 \mathrm{~h}\end{array}$ & 207.000 & 204.000 & 120.000 & 140.000 & 160.000 & 180.000 & 200.000 \\
\hline
\end{tabular}

Wir erhalten folgende Ergebnisse, die auch in einer analytischen Prüfung gegenüber Änderungen der Gewichtungsfunktion robust sind..$^{30}$

- Der wahrgenommene erwartete Nutzen zusätzlichen Sorgfaltsaufwands (mehr Arbeitsstunden) wird unterschätzt, die Kosten hingegen nicht, da sie mit Sicherheit anfallen (zur Erinnerung: $\omega(p=1)=1$ ). Dies dämpft die Sorgfaltsanreize.

- Bei Gefährdungshaftung wählt der Abschlussprüfer zwingend ein Sorgfaltsniveau, was geringer ist als das gesellschaftlich wünschenswerte (in unserem Beispiel wählt er 500h). Bei Verschuldenshaftung werden die Sorgfaltsanreize ebenfalls tendenziell gedämpft, allerdings nicht zwingend. So wird der Prüfer in unserem Beispiel weiterhin den gesetzlichen Standard einhalten.

Der Sicherheitseffekt gibt dem Prüfer einen zusätzlichen Anreiz, den gesetzlichen Standard zu wählen, da er dann mit Sicherheit weiß, nicht zu haften. Dies ist eine gute

30 Siehe Bigus (2006b). 

sein?

Nachricht, sofern der gesetzliche Sorgfaltsstandard »richtig « gewählt wurde und dem gesellschaftlichen Optimum entspricht. Wird er zu hoch oder zu niedrig angesetzt, hat der Prüfer natürlich ebenfalls stärkere Anreize, den falsch gewählten Standard zu wählen.

\subsection{Der Effekt der Wahrscheinlichkeitsgewichtungsfunktion auf die Sorgfaltsanreize des Abschlussprüfers bei unpräzise definiertem Sorgfaltsstandard}

Zuweilen ist der gesetzliche Standard unpräzise definiert. Bei einem präzisen Standard ist dem potentiellen Schädiger ex-ante ganz genau die »rote Linie« bekannt, die fahrlässiges und nicht fahrlässiges Verhalten voneinander trennt. So ist jedem Autofahrer bewusst, dass die Geschwindigkeitsgrenze, die fahrlässiges Verhalten in einer geschlossenen Ortschaft markiert, $50 \mathrm{~km} / \mathrm{h}$ beträgt. In der Praxis der Abschlussprüferhaftung ist die Linie häufig weniger trennscharf. Im Zweifel muss ein Gericht ex-post feststellen, ob die im Verkehr erforderliche Sorgfalt aufgewendet wurde. ${ }^{31}$ Wie unpräzise ein Standard ist, hängt von vielen Faktoren ab, etwa von der Neuartigkeit des Rechtsgebiets und vom Grad der juristischen Durchdringung im Schrifttum und im Richterrecht, zudem von der Wahrscheinlichkeit, dass Gerichte in Folge mangelnden Expertenwissens und/oder mangelnder Unabhängigkeit die gesellschaftlich optimale Lösung verfehlen. Ex-ante, d.h. im Zeitpunkt der Prüfung, gibt es damit einen »Graubereich «, den die Abbildung 3 illustriert.

\section{Abb. 3: Unpräziser Prüfungsstandard}

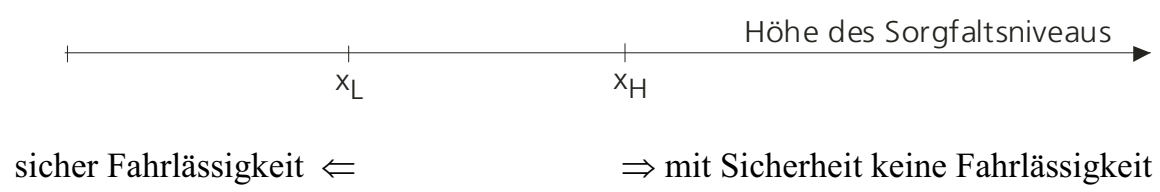

Wird das Sorgfaltsniveau $x_{L}$ nicht überschritten, so kann der Wirtschaftsprüfer $e x$ ante mit Sicherheit davon ausgehen, dass das Gericht Fahrlässigkeit feststellen wird und er Schadenersatz leisten muss. Wird zumindest das Niveau $x_{H}$ erreicht, ist eindeutig die erforderliche Sorgfalt aufgewendet worden. Zwischen diesen Sorgfaltsniveaus, d.h. im Bereich $x_{L}<x<x_{H}$, ist es im Prüfungszeitpunkt unsicher, wie das Gericht entscheiden wird. Es sei aber unterstellt, das effiziente Sorgfaltsniveau (das erst im Nachhinein exakt festgestellt wird) liege im »Graubereich $«\left(x_{L}<x *<x_{H}\right){ }^{32}$

In der rechtsökonomischen Literatur ist bekannt, dass die Existenz eines unpräzisen Standards die Sorgfaltsanreize des Schädigers tendenziell erhöht, oftmals sogar über

31 Zur Analyse unpräziser Prüfungsstandards im Lichte anderer Fragestellungen siehe auch Crasswell/Calfee (1986), Ewert (1999) und Bigus/Schäfer (2007).

32 Diese Annahme erscheint plausibel, wenn man annimmt, dass Gerichte bei der Feststellung der »im Verkehr erforderlichen Sorgfalt« das effiziente Sorgfaltsniveau anstreben, allerdings wegen mangelnder Information den Sorgfaltsmaßstab mal höher, mal niedriger als das effiziente Niveau ansetzen. 
das gesellschaftlich wünschenswerte Maß hinaus. ${ }^{33}$ Ein höheres Sorgfaltsniveau verringert nicht nur die Wahrscheinlichkeit eines Schadens, sondern - anders als bei präzise definiertem gesetzlichen Sorgfaltsstandard - grundsätzlich auch die Wahrscheinlichkeit, dass das Gericht im Nachhinein auf Fahrlässigkeit erkennt. Auch wenn der Prüfer aus Sicht der Anteilseigner daher tendenziell zu scharf prüfen und das gesellschaftlich optimale Niveau $x *$ überschreiten wird, wird er dennoch nach der klassischen Theorie häufig unter dem Sorgfaltsniveau $x_{H}$ bleiben. Eine Haftung ist daher mit einer gewissen Wahrscheinlichkeit möglich.

Wie wirkt nun der Sicherheitseffekt, den die Wahrscheinlichkeitsgewichtungsfunktion der Prospect Theory impliziert? Der Sicherheitseffekt gibt dem Prüfer zusätzliche Anreize, sorgfältig zu arbeiten, wenn eine ansonsten bestehende geringe Haftungswahrscheinlichkeit auf Null abgesenkt werden kann. Dies ist bei präzisen Sorgfaltsstandard eine zusätzliche Motivation, den gesetzlichen Standard einzuhalten und aus ökonomischer Sicht eine gute Nachricht, sofern der gesetzliche Standard das gesellschaftliche Optimum darstellt.

Bei unpräzise definiertem Standard kann dies aber dazu führen, dass der Schädiger einen Anreiz verspürt, das Sorgfaltsniveau $x_{H}$ zu wählen, um einer Haftung mit Sicherheit zu entgehen. Damit ist er noch weiter vom gesellschaftlich optimalen Niveau $x^{*}$ entfernt. Je unpräziser der Fahrlässigkeitsmaßstab ist, desto eher besteht aber auch die Gefahr, dass der potentielle Schädiger einen übermäßigen Schadensvermeidungsaufwand betreibt und sich somit noch mehr von dem gesellschaftlich wünschenswerten Optimum entfernt. ${ }^{34}$ Es ist sicherlich kein Zufall, dass wir in den USA die Koexistenz sehr scharfer Haftungsregeln und sehr präzise definierter Rechnungslegungs- und Prüfungsregeln beobachten.

Festzuhalten ist zudem ein weiterer Befund: Den Sicherheitseffekt kann es nur mit einer Verschuldenshaftung geben, nicht aber mit einer Gefährdungshaftung, weil hier immer eine gewisse, noch so kleine Wahrscheinlichkeit einer Haftung besteht.

\section{Diskussion}

Wir haben bei der Analyse der Abschlussprüferhaftung unterstellt, der Prüfer handele im Sinne der Prospect Theory. Ob die Prospect Theory tatsächlich relevant ist, ist noch umstritten. Kritisiert wird, dass die Prospect Theory primär auf den Ergebnissen einer Vielzahl von Laborexperimenten basiert, die vornehmlich - aber nicht ausschließlich - mit Studenten durchgeführt worden sind (die Akquise von Entscheidern aus der Berufspraxis scheitert häufig schon aus Kostengründen). Oft wird kritisiert, dass die Entscheidungslage in einem Labor wenig mit tatsächlichen Situationen zu tun habe, auch wenn man finanzielle Anreize setzt und um ein möglichst neutrales Verfahren bemüht ist. Auch andere Aspekte des Untersuchungsdesigns früherer Studien werden teilweise kritisiert. Damit werden auch die grundlegenden Charakteristika der Prospect Theory

33 Siehe Crasswell/Calfee (1986) und Bigus (2007).

34 Im Rahmen der Abschlussprüfung kann man vermuten, dass der Sorgfaltsstandard bei der Prüfung von IFRS-Abschlüssen weniger klar ist als bei der Prüfung von HGB-Abschlüssen. 

sein?

in Frage gestellt. Einige Beiträge behaupten sogar, dass die Erwartungsnutzentheorie besser geeignet sei, um tatsächliches Entscheidungsverhalten zu erklären. ${ }^{35}$ Allerdings sind diese Beiträge in der Minderheit.

Kurzum: Man weiß derzeit nicht mit Sicherheit, nach welchem Modell Individuen Entscheidungen treffen. Für Individualentscheidungen scheinen mehr Befunde für die Prospect Theory als für die Erwartungsnutzentheorie zu sprechen. Es ist nicht absehbar, wann diese empirische Frage endgültig geklärt ist. Dies sollte aber die eher theoretisch und normativ ausgerichtete rechtsökonomische Forschung nicht davon abhalten, auch den Fall beschränkt rationalen Verhaltens eingehender zu betrachten. Die weitaus meisten rechtsökonomischen und auch wirtschaftswissenschaftlichen Beiträge unterstellen immer noch Individuen, die nach der Erwartungsnutzentheorie entscheiden.

Die Prospect Theory stellt primär auf Individualentscheidungen ab. Institutionelle Akteure, etwa Unternehmen, etwa Prüfungsgesellschaften, werden versucht sein, Mechanismen zu verankern, die eine rationale Entscheidungsfindung der Mitarbeiter unterstützen, etwa im Wege strukturierter Entscheidungsprozesse - auch mit Hilfe von Gruppenentscheidungen. Unternehmen werden rationaler Weise auch aus der detaillierten Analyse früherer Fehlentscheidungen lernen. Allerdings ist die empirische Evidenz für die Vermutung, dass Institutionen rationaler als Individuen handeln würden, nicht eindeutig. ${ }^{36}$ Rabin (1998) betont zudem, dass die Lerneffekte in der Realität doch deutlich begrenzter sind als Ökonomen erwarten. Dennoch ist zu vermuten, dass beschränkt rationales Verhalten eher bei individuellen als bei institutionellen Schädigern auftritt, und auch umso eher, je geringer die Lerneffekte sind, etwa bei eher seltenen Schadensfällen. Kurzum, wir sollten beschränkt rationales Verhalten eher im Kontext der individuellen Haftung im Straßenverkehr als bei der Produkthaftung von Unternehmen der Konsumgüterindustrie erwarten.

\section{Zusammenfassung}

Dieser Beitrag untersucht, inwieweit die Ergebnisse der ökonomischen Analyse des Deliktsrechts Bestand haben, wenn man unterstellt, dass der potentielle Schädiger beschränkt rational im Sinne der Prospect Theory handelt. Die Prospect Theory stellt ein alternatives Entscheidungsmodell zur klassischen Erwartungsnutzentheorie dar, die in der Wirtschaftswissenschaft und auch im Bereich Law and Economics noch vorherrschend ist. Die meisten Studien lassen den Schluss zu, dass die Prospect Theory tatsächliches Entscheidungsverhalten offenbar besser abbilden kann. Dabei wurden die Wirkungen zweier Elemente der Prospect Theory separat untersucht.

Verlustaversion. Verluste werden stärker gewichtet als betragsmäßig gleich hohe Gewinne. Wenn sowohl Schadensvermeidungsaufwand - im Kontext der Abschlussprüfung: Prüfungsaufwand - als auch der potentielle Ersatz späterer Schäden als Verlust angesehen wird, induzieren sowohl Gefährdungshaftung als auch eine korrekt spe-

35 Siehe etwa Myagkov / Plott (1997) und List (2004).

36 Siehe etwa im Kapitalmarktkontext Odean (1998). 
zifizierte Verschuldenshaftung tendenziell ein gesellschaftlich optimales Prüfungsniveau (jedenfalls in einem Kontext der Abschlussprüferhaftung, der dem US-amerikanischem weitgehend entspricht). Wird hingegen lediglich der künftige Schadenersatz als Verlust angesehen, wird eine Gefährdungshaftung den Wirtschaftsprüfer zu übermäßigem Sorgfaltsaufwand anhalten. Bei einer Verschuldenshaftung sind gesellschaftlich optimale Lösungen zumindest möglich, sie ist daher vorzuziehen. Dies steht im Kontrast zur klassischen ökonomischen Analyse des Deliktsrechts, wonach die Verschuldenshaftung keine besseren Anreize zur Schadensvermeidung vermitteln kann.

Wahrscheinlichkeitsgewichtung: Niedrige (Schadens-)Wahrscheinlichkeiten werden überschätzte, große Wahrscheinlichkeiten hingegen unterschätzt. Der Übergang von einem fast sicheren Ereignis (99\% Wahrscheinlichkeit) zur Sicherheit (100\%), wird deutlich stärker als mit einem Prozentpunkt gewichtet. Dies bezeichnet man auch als Sicherheitseffekt. Die Gewichtung der Wahrscheinlichkeiten führt tendenziell dazu, dass sich ein Prüfer bei einer Gefährdungshaftung zu wenig anstrengt, bei einer Verschuldenshaftung mit präzisem Standard ist dennoch eine gesellschaftlich optimale Lösung erzielbar. Der Sicherheitseffekt besteht nur bei Verschuldenshaftung, bei der man eine Haftung ausschließen kann, sofern ein gewisser Sorgfaltsstandard eingehalten wurde. Bei der Gefährdungshaftung kann dies nicht ausgeschlossen werden. Der Sicherheitseffekt gibt bei präzise definiertem Sorgfaltsstandard zusätzliche Anreize, diesen zu wählen. Je unpräziser allerdings der gesetzliche Standard ex-ante aus Sicht des Prüfers ist, desto eher besteht die Gefahr, alle Anstrengungen zu unternehmen, um eine Haftung auszuschließen. Damit wählt der Abschlussprüfer ein zu hohes Sorgfaltsniveau - zumindest aus Sicht der Anteilseigner, die ihn bezahlen müssen. Dieser Fall der übermäßigen Sorgfalt ist eher bei der Prüfung von IFRS- als bei HGB-Abschlüssen zu vermuten, da die Rechtslage hier noch unklarer ist.

Insgesamt induziert eine korrekt spezifizierte Verschuldenshaftung eher gesellschaftlich wünschenswerte Sorgfaltsanreize als eine Gefährdungshaftung, wenn man beschränkt rationales Verhalten des Abschlussprüfers unterstellt. Die Ergebnisse dieses Beitrags mögen daher auch erklären, weshalb die Abschlussprüfer in den westlichen Ländern ausschließlich für Verschulden haften. Allerdings habe ich auch die Grenzen der Prospect Theory dargelegt. Zusätzlich ist zu beachten, dass die Wirkung einzelner Charakteristika separat untersucht wurde. Eine simultane Wirkungsanalyse muss das Ziel künftiger Forschungsanstrengungen sein. Wünschenswert ist auch eine empirische Validierung der hier gemachten Aussagen.

\section{Literaturverzeichnis}

Allais, M. (1953): Le comportement de l'homme rationel devant le risque: Critique des postulats et axiomes de l'école américaine, Econometrica, Vol. 21, 503-546.

Bigus, J. (2006a) : Reputation und Wirtschaftsprüferhaftung, Betriebswirtschaftliche Forschung und Praxis, 58. Jg., 22-41.

Bigus, J. (2006b) : Auditors' Liability and Prospect Theory, Arbeitspapier Universität Osnabrück, März 2006. 

sein?

Bigus, J. (2007): Die Sorgfaltsanreize des Wirtschaftsprüfers bei beschränkter Haftung, Zeitschrift für betriebswirtschaftliche Forschung, 59. Jg., 61-86.

Bigus, J. / Schäfer, H.-B. (2007): Die Haftung des Wirtschaftsprüfers am Primärmarkt und am Sekundärmarkt - eine rechtsökonomische Analyse, Zeitschrift für Betriebswirtschaft.

Bitz, M. (1981) : Entscheidungstheorie, München 1981, 77. Jg., 19-49.

Cooter, R. / Ulen, Th. (2004) : Law and Economics, 4. Aufl., Boston et al. 2004.

Craswell, R./ Calfee, J.E. (1986): Deterrence and Uncertain Legal Standards, Journal of Law, Economics, and Organization, Vol. 2, 279-303.

Dye, R. (1993): Auditor Standards, Legal Liability, and Auditor Wealth, Journal of Political Economy, Vol. 101, 887-914.

Eide, E. (2006): Accident Liability with Rank Dependent Expected Utility, Arbeitspapier Universität Oslo, Juni 2006, in diesem Band, 159-170.

Ewert, R. (1999): Auditor Liability and the Precision of Auditing Standards, Journal of Institutional and Theoretical Economics, Vol. 155, 181-206.

Ganuza, J.J. / Gomez, F. (2005): Should We Trust the Gatekeepers? Auditors' and Lawyers' Liability for Clients' Misconduct, Working Paper, Universität Pompeu Fabra, Barcelona, Februar 2005.

Hillegeist, St. A. (1999): Financial Reporting and Auditing under Alternative Damage Apportionment Rules, The Accounting Review, Vol. 74, 347-369.

Kahneman, D. / Tversky, A. (1979): Prospect Theory: An Analysis of Decision under Risk, Econometrica, Vol. 47, 263-291.

List, J. (2004): Notes and Comments. Neoclassical Theory versus Prospect Theory: Evidence from the Marketplace, Econometrica, Vol. 72, 615-625.

McCrimmon, K.R./ Larsson, S. (1979): Utility Theory: Axioms versus Paradoxes, in: Allais, M./ Hagen, O. (Hrsg.): The Expected Utility Hypothesis and the Allais Paradox, Holland, Dordrecht, 333-409.

Myagkov, M. / Plott, Ch. (1997): Exchange Economies and Loss Exposure: Experiments exploring Prospect Theory and Competitive Equilibria in Market Environments, American Economic Review, Vol. 87, 801-828.

Odean, T. (1998): Volume, Volatility, Price and Profit when all Trades are above Average, Journal of Finance, Vol. 53, 1775-1798.

Prelec, D. (1998): The Probability Weighting Function, Econometrica, Vol. 66, $497-$ 528.

Quiggin, J. (1982): A Theory of Anticipated Utility, Journal of Economic Behaviour and Organisation, Vol. 3, 323-343.

Rabin, M. (1998): Psychology and Economics, Journal of Economic Literature, Vol. 36, 11-46.

Radhakrishnan, S. (1999): Investors' Recovery Friction and Auditor Liability Rules, The Accounting Review, Vol. 74, 225-240.

Schäfer, H.-B. (2004): Efficient Third Party Liability of Auditors in Tort Law and Contract Law, Supreme Court Economic Review, Vol. 4.

Schäfer, H.-B. / Ott, C. (2005): Lehrbuch der ökonomischen Analyse des Zivilrechts, 4. Aufl., Berlin et al. 
Schmeidler, D. (1989): Subjective Probability and Expected Utility without Additivity, Econometrica, Vol. 57, 571-587.

Schwartz, R. (1997): Auditors' Liability, Vague Due Care, and Auditing Standards, Review of Quantitative Finance and Accounting, Vol. 11, 183-207.

Segal, U. (1987): Some remarks on Quiggin's Anticipated Utility, Journal of Economic Behaviour and Organisation, Vol. 8, 145-154.

Shavell, S. (1987): Economic Analysis of Accident Law, Cambridge (Mass.).

Shavell, S. (2004): Foundations of Economic Analysis of Law, Cambridge (Mass.).

Sunstein, C.R. (2000): Behavioral Law and Economics, Cambridge (Mass.).

Sunstein, C.R. / Kahneman, D. / Schkade, D. (2000): Assessing Punitive Damages, in: Sunstein, C.R. (Hrsg.): Behavioral Law and Economics, Cambridge (Mass.), 232258.

Teitelbaum, J.C. (2006): A Unilateral Accident Model under Ambiguity, Working Paper Cornell University, Januar 2006, erscheint in Journal of Legal Studies.

Tversky, A. / Kahneman, D. (1992): Advances in Prospect Theory: Cumulative Representation of Uncertainty, Journal of Risk and Uncertainty, Vol. 5, 297-323.

Zhang, Ping / Thoman, L. (1999): Pre-Trial Settlement and the Value of Audits, The Accounting Review, Vol. 74, 473-491. 